\title{
INTERNAÇÕES HOSPITALARES NO RIO GRANDE DO SUL*
}

\author{
Stela Nazareth Meneghell', Teresa Borget Armani', Roger dos Santos Rosa', \\ Lenine de Carvalho', Alunos do XX CESP²
}

\begin{abstract}
Este é um estudo descritivo, realizado como trabalho de conclusão do XX Curso de Especialização em Saúde Pública/Escola de Saúde Pública/Secretaria da Saúde e do Meio-Ambiente (XX CESP/ESP/SSMA), Departamento de Medicina Social/ Universidade Federal do Rio Grande do Sul (DMS/UFRGS) e Escola Nacional de Saúde Pública (ENSP/FIOCRUZ). Analisaram-se as 817.244 internaçסes hospitalares ocorridas no Rio Grande do Sul em 1995 através do SUS (8,6 hospitalizaçסes $/ 100$ habitantes). Os grupos que mais demandaram os serviços foram as mulheres, os menores de um ano e os idosos. As principais causas de internaçåo foram: doenças respiratórias, parto e doenças do aparelho circulatorio.
\end{abstract}

\section{Introdução}

Neste artigo apresenta-se o resumo do trabalho de conclusão do XX Curso de Especialização em Saúde Pública (CESP/96), realizado na Escola de Saúde Pública/Secretaria da Saúde e do Meio-Ambiente do Rio Grande do Sul (ESP/SSMA/RS), em convênio com a Universidade Federal do Rio Grande do Sul/ Faculdade de Medicina/Departamento de Medicina Social (UFRGS/FAMED/DMS) e com a FIOCRUZ/Escola Nacional de Saúde Pública. Descreve-se o perfil da morbidade hospitalar no Estado do Rio Grande do Sul em 1995.

Nos últimos anos, tem-se identificado carência de estudos e pesquisas sobre as doenças que mais atingem a população no Rio Grande do Sul, quer seja no nível ambulatorial como no nível hospitalar, ao passo que os dados de mortalidade têm sido exaustivamente analisados. Com base nessa constatação, $o$ CESP/1996 buscou contemplar a necessidade de formar sanitaristas para atuar e revigorar as açôes de saúde no nível das Delegacias Regionais das Saúde(DRS), enfatizando o instrumental epidemiológico e a análise de dados de morbidade.

OCurso foi reformulado para adequarse a esses objetivos. Trabalhou-se no formato de oficinas, com dados dos serviços, sem perder, porém, o espaço teórico-conceitual de produção de conhecimentos, compatibilizando a relação acadêmica com os serviços numa constante aproximação entre o "saber" e o

*Estudo epidemiológioo de metodologia coletiva de ahunos e professores do XX Curso de Especializaçáo em Saúde Pública.

${ }^{1}$ Professores e pesquisadores, orientadores do trabalho.

${ }^{2}$ Auxiliares de Pesquisa.

Escola de Saúde Pública. Av. Ipiranga 6311, Porto Alegne - Rio Grande do Sul - 90610-001. 
"saber fazer". O objetivo principal deste trabalho foi servir como campo de prática para os alunos do Curso e, ao mesmo tempo, atender à necessidade de usar as informaçóes do extenso banco de dados das Autorizações de Internação Hospitalar (AIH/SUS), cuja finalidade tem sido quase que exclusivamente voltada ao pagamento das contas hospitalares.

Salienta-se, ainda, um aspecto fundamental: a metodologia desenvolvida nessa investigação. No Brasil, a forma predominante de organização do trabalho nos cursos de pósgraduação tem sido a individual; por outro lado, acredita-se que o ensino superior não deve apenas preparar indivíduos, mas grupos de pesquisa $^{1}$. Muito se tem discutido acerca da produção coletiva de conhecimento. Não só ela é mais econômica, reduzindo custos e potencializando esforços mas, principalmente nesse tipo de trabalho, experimenta-se uma nova prática, aprendendo-se a conviver com as competiçōes, os atritos interpessoais, a hierarquia de competências, os desentendimentos e os diferentes pontos de vista.

No caso deste trabalho, esse processo caracterizou-se sobretudo pela flexibilidade. $O$ planejamento inicial serviu apenas como premissa de direcionamento, adotando-se uma metodologia de construção/reconstrução durante todos os momentos.

Acredita-se que os movimentos de vai e vem, acertos e erros, típicos dessa metodologia, fazem parte do processo de conhecimento da realidade. Sem dispor de uma carta pré-fabricada de soluçóes. O mapeamento do caminho e o próprio mapa ocorrem simultaneamente. Espera-se que essa metodologia de trabalho permaneça incorporada no "que e como fazer" desses sanitaristas. Espera-se também continuála e aperfeiçoá-la em outras pesquişas de grande grupo, em outros mutiróes de saúde coletiva.

"A vida inventa! A gente principia as coisas no não saber por que, e desde aí perde o poder de continuação - porque a vida é mutirão de todos, por todos remexida e temperadia”.

Guimarães Rosa

\section{Morbidade nas populações}

\subsection{Estudos de morbidade}

O processo saúde/doença, do ponto de vista epidemiológico, é a síntese do conjunto de determinações que acontecem em umă sociedade, produzindo riscos ou potencialidades características nos diferentes grupos sociais, que se manifestam na forma de perfis ou padrōes de doença ou de saúde ${ }^{2}$. A doença é produto direto ou indireto das condições gerais em que se desenvolve essa sociedade e das condiçóes particulares de desenvolvimento de uma determinada classe social. Tem-se tentado dimensionar a saúde/doença nas populações através de indicadores de saúde, entre os quais os de morbidade e de mortalidade. Morbidade é o termo utilizado para expressar a presença de doença ou condição patológica. As estatísticas de morbidade são um poderoso indicador da saúde de uma comunidade e, como tal, podem auxiliar no planejamento, na programação, no controle e na avaliação das açốes de saúde ${ }^{3}$.

A morbidade compreende múltiplos aspectos, desde a percepção da população acerca do normal/patológico até a definição do que é um caso clínico. Ela traduz a situação saúde/ doença de um grupo social determinado, envolvendo aspectos biológicos, culturais, antropológicos, econômicos e psicológicos.

Travassos e cols. sugerem que há um acentuado componente social tanto na morbidade quanto no uso de serviços de saúdes. A ưilização de serviços de saúde está vinculada às necessidades da população. No entanto, essas necessidades não são homogêneas entre os grupos sociais. As chances de adoecer e de morrer são influenciadas pela posição social e pela experiência de vida dos indivíduos.

A oferta dos serviços obedece às leis do mercado, que tende a alocá-los conforme a capacidade de pagamento, prejudicando especialmente os mais desfavorecidos e doentes ${ }^{5}$. Carvalho e cols. 
referem que "a utilizaf̧ão de serviços de saúde parece expressar, não necessariamente a demanda potencial real por esses servicos entre a popula cão brasileira, mas sim o resultado de todo um processo que funciona como uma espécie de filtro social, onde aspectos culturais, institucionais $e$ socioeconômicos distintos somam-se às condif̧ôes de desigualdade regional"s.

Entre os fatores que interferem nos índices de morbidade de uma região pode-se citar: a disponibilidade e acessibilidade aos serviços de saúde; a percepção das pessoas à saúde/doença; a estrutura demográfica da população. As taxas de morbidade variarão de acordo com a existência e a proximidade dos serviços de saúde. Assim, a procura por serviços de saúde é mais elevada nas regióes urbanas do que nas rurais ${ }^{7}$, refletindo maior dificuldade de acesso e menor oferta de serviços nessas últimas.

"A distmbuição da rede de servicos de saúde do Brasil é profundamente desigual, quando se consideram as distintas regióes do pais, as áreas urbanas ou rurais, as regióes ricas e pobres das cidades, determinando, junto com outros fatores socioeconómicos e culturais uma extrema desigualdade na oferta e no acesso aos servifos de saride pelas diferentes populayốes e estratos sociais".

Nem todas as pessoas que adoecem em uma determinada regiáo buscam os serviços de saúde. Há uma parcela que não chega aos serviços pelas mais variadas razōes, que incluem desde baixa gravidade da doença até barreiras econômico-culturais. Entre os que buscam os serviços, alguns são tratados em nível ambulatorial, alguns realizam exames suplementares e apenas uma parcela, supostamente a mais grave, é internada em hospitais.

A morbidade tem sido abordada sob diferentes aspectos e de forma específica, de acordo com o tipo de estudo e interesse dos pesquisadores e das instituições.

Um dos maiores estudos de morbidade foi preparado em conjunto pela Organização Mundial de Saúde e pelo Banco Mundial para o Relatório sobre o Desenvolvimento Mundial/
1993: Investindo em Saúde. O Relatório quantificou o impacto global da doença em termos de perda de vida saudável, somando a perda de vida por morte prematura e por incapacidade. Uma das características mais originais desse estudo foi a utilização de um indicador misto de morbi-mortalidade denominado disability-adjusted life-year (DALYs) ou Anos de Vida Ajustados por Incapacidade (AVAIs) ${ }^{9}$.

Num estudo realizado na década de setenta - International Collaborative Study on Health Care - a morbidade referida variou de 27-35\% no Canadá, 26-32\% nos Estados Unidos, 35\% em Buenos Aires, 32\% em Londres, $42 \%$ na Polônia e $36-46 \%$ na Iugosláviá.

No Brasil, a primeira grande pesquisa sobre morbidade referida foi realizada no âmbito da Pesquisa Nacional por Amostra de Domicílios (PNAD/FIBGE). A morbidade foi investigada nas Pesquisas Nacionais por Amostra de Domicílios (PNADs) a partir da referência a algum problema de saúde nos quinze dias anteriores à entrevista. $\mathrm{Na}$ PNAD/ 81 as taxas de morbidade variaram de $3,8 \% \mathrm{em}$ Belém a 9,6\% em Belo Horizonte ${ }^{6}$ Na PNAD/ 86 , o percentual situou-se em $17 \%$, oscilando de $14 \%$ na Região Norte a $18 \%$ na Sudeste; além disso, a morbidade referida foi maior nas regiōes urbanas e entre as mulheres?.

Pinto e cols. efetuaram estudo sobre morbidade hospitalar na rede contratada do INAMPS entre os anos de 1984 e $1986^{10}$. Nessa pesquisa, os autores compararam os dados de morbidade obtidos através do Sistema de Informações Hospitalares do Sistema Único de Saúde (SIH/SUS) com os dados de mortalidade obtidos pelo Sistema de Informaçóes de Mortalidade (SIM), numa tentativa de averigüiar a fidedignidade desses distintos sistemas de informação. Mostraram também que é possível utilizar o SIH/SUS para obter informaçōes de natureza epidemiológica. Segundo os autores, as causas de internação mais freqüentes conforme os 17 grupos de causas da Classificação Internacional de Doenças (CID) foram: em 
primeiro lugar, as complicaçóes da gravidez, parto e puerpério, seguidas pelas doenças do aparelho respiratório, doenças do aparelho circulatório, doenças infecciosas e parasitárias e doenças do aparelho geniturinário.

Em um estudo sobre morbidade hospitalar, no município de Cubatáo (SP), foram identificadas as principais patologias da rede hospitalar, com a finalidade de subsidiar a atuação dos serviços de saúde do município. Esse estudo mostrou que as principais causas de internação no período de julho/1983 a junho/1984 foram as complicaçôes da gravidez, do parto e do puerpério e doenças do aparelho respiratório ${ }^{11}$.

Siméant propôs uma metodologia simples e de baixo custo para a avaliação da capacidade resolutiva em estabelecimentos de nível primário de atenção à saúde, no Chile ${ }^{12}$. $\mathrm{O}$ autor encontrou diferenças na estrutura da morbidade de acordo com o tipo de estabelecimento - posto de saúde, consultório geral rural e consultónio geral anexo ao hospital.

O trabalho desenvolvido por Yazlle Rocha, no município de Ribeirão Preto (SP), teve como objetivo apresentar os padröes de morbidade em assistência primária ${ }^{13}$. A maior demanda foi constituída por crianças, mulheres e idosos. Os diagnósticos mais frequientes foram as doenças infecciosas e parasitárias, doenças do aparelho respiratório, inflamaçôes dos olhos e ouvidos, doenças da pele e do tecido subcutâneo e doenças do aparelho genitourinário.

$O$ objetivo da pesquisa realizada por Radaelli e cols., na Unidade Sanitária Murialdo de Porto Alegre (RS), foi conhecer os motivos de consulta mais freqüentes $\mathrm{em}$ um serviço de medicina comunitária da rede estadual ${ }^{14}$. Nessa pesquisa, o sexo feminino ocupou $67 \%$ do total do atendimento. A composição etária de maior ocorrência foi de crianças de até 10 anos (37\%) e mulheres em idade fértil (21\%). O motivo principal da procura do serviço foi a renovação de receita médica e pedidos de medicamento $(9,3 \%)$. Os diagnósticos mais freqüentes foram: hipertensão arterial sistêmica $(8,8 \%)$, afecçōes das vias aéreas superiores $(7,8 \%)$ e busca de imunizações $(5,5 \%)$.
Carvalho e cols. realizaram estudos sobre morbidade referida e utilizaçáo de consulta médica no Estado da Bahia no período de 1982 a $1986^{\circ}$. Os resultados mostraram que a morbidade variou de $19,2 \%$ a $40,3 \%$. As taxas de utilização de consulta médica nos quinze dias anteriores à entrevista oscilaram de $4,9 \%$ a $15,2 \%$, as maiores taxas ocorrendo nas áreas urbanas e as menores nas áreas rurais.

Uma pesquisa domiciliar sobre o perfil de morbidade populacional e o padrão de utilização de serviços de saúde em localidades urbanas brasileiras evidenciou que o grupo de menores de um ano e a populaçâo idosa apresentaram as maiores prevalências de problemas de saúde e freqüentemente procuraram mais os serviços de saúde ${ }^{15}$.

Travassos e cols. desenvolveram uma investigaçáo com o objetivo de avaliar a eqüiidade no uso de serviços de saúde no país, com dados oriundos da $\mathrm{PNAD} / 89^{4}$. Constataram que a morbidade estava desproporcionalmente distribuida entre grupos de renda, concentrandose nos mais pobres, que menos utilizavam os serviços de saúde.

\subsection{Sistemas de Informação e Morbidade}

Os dados sobre morbidade no Brasil são muito limitados, não existindo até o momento um sistema nacional que se proponha a avaliar a morbidade geral. As informaçōes colhidas de forma direta e para todo o país são sobre as doenças passíveis de notificação compulsória e as comunicações de acidente de trabalho. Outras informaçóes sobre morbidade podem ser obtidas por meio da análise do Sistema de Informações da Demanda Ambulatorial (SIA/SUS) ou do Sistema de Informações Hospitalares (SIH/ SUS).

A Autorização de Internação Hospitalar (AIH) é o documento hábil para identificar o paciente e os serviços prestados sob regime de internação hospitalar. Fornece informaçōes para o gerenciamento do sistema $e$, através dele, os hospitais, profissionais e serviços auxiliares de diagnose e terapia receberão pelos serviços prestados ao usuário ${ }^{16}$. Ao utilizar-se tal instrumento como fonte de dados para um estudo de 
morbidade hospitalar, devem ser considerados os aspectos de fidedignidade e validade das informações originalmente destinadas ao uso administrativo. Contudo, apesar da AIH não ser completamente imune às distorçóes do sistema, o fato de existir um registro sistemático de doenças, vigente em todo o país e ao qual se pode ter fácil acesso, revela um valioso campo de investigação.

Poucos estudos utilizaram o SIH/SUS como fonte para análise da morbidade até o presente momento. $\mathrm{O}$ primeiro estudo de morbidade hospitalar da rede contratada do INAMPS já foi citado anteriormente ${ }^{10}$.

Buss realizou um estudo que examinava algumas variáveis da assistência hospitalar prestada pelo SUS no período de 1984 a 1991, centrando-se nos diagnósticos de alta por grupos de causas e nos custos assistenciais?.

Porém, cada vez mais administradores e epidemiologistas têm ressaltado a importância do uso de dados administrativos não só para avaliar a qualidade dos serviços médicos, como também para traçar perfis de ocorrência de agravos. As vantagens de se analisar dados secundários incluem menores custos e tempo de pesquisa porque, apesar das conhecidas limitaçōes, eles são um meio de estudar grandes volumes de dados, apontar problemas que necessitem ser observados e gerar hipóteses para mais tarde serem testadas com dados primários ${ }^{17}$.

\section{Considerações metodológicas}

Este é um estudo descritivo e exploratório que utilizou dados secundários. Realizou-se um esforço de sistematização, trazendo à luz novos elementos a outros profissionais de saúde que de outra forma não teriam como acessá-los. São dados públicos; porém em tal volume que exigem um esforço analítico nem sempre disponível no dia-a-dia dos trabalhadores de saúde.

As populaçóes de cada DRS utilizadas para coeficientes foram estimadas pelo DATASUS/Fundaçáo Nacional de Saúde (FNS), com base no Censo de 1991.
Os dados de morbidade são procedentes do Sistema de Informaçóes Hospitalares do SUS (SIH-SUS) e referentes ao ano de 1995, cuja fonte é a Autorização de Internação Hospitalar (AIH). Foram analisadas 817.244 internaçốes consideradas normais (aquelas de início de internação) e excluídas 10.064 de longa permanência "Fora de Possibilidade Terapêutica"(FTP), bem como as AIHs de continuação (quando esgotado o espaço de preeenchimento da AIH normal é necessário uma continuação), pois essa última possui a mesma numeraçáo da AIH normal que lhe deu origem, não alterando o número de internaçôes hospitalares. As AIHs de longa permanência/ FPT podem referir-se também a internaçóes de anos anteriores, elevando o número total de internações do ano em estudo.

Os dados de morbidade foram coletados no banco de AIHs e apresentados na forma de CD-ROM, elaborado pela Fundação Nacional de Saúde (FNS). A análise foi realizada através do Programa TAB 16.1.18.

Os indicadores trabalhados foram:

- coeficiente de morbidade hospitalar/DRS: colocou-se no numerador o número de AIHs/ DRS em relação à população da Delegacia;

- distribuiçáo percentual da morbidade segundo grupo etário: selecionou-se no numerador o número de AIHs conforme cinco grupos etários ( $<1$ ano; 1 a $4 ; 5$ a $14 ; 15$ a $49 ; 50$ e + anos) em relação ao total das internaçóes;

- coeficiente de morbidade por grupo etário/ RS: relacionou-se no numerador o número de $\mathrm{AIH}$ s por grupo etário em relação à população nas diferentes idades;

- distribuição percentual da morbidade segundo 17 grupos de causa da CID: distribuiu-se no numerador o número de internaçóes segundo causa em relação ao total de AIHs;

- distribuição percentual das principais causas de internaçáo (CID-3 dígitos): listou-se as principais causas de internação em relação ao total. 
comparação da morbidade percentual (SIHSUS) e mortalidade proporcional (SIM), segundo grupo de causas ( 17 seçóes da CID).

\section{Morbidade hospitalar da população do Rio Grande do Sul}

No Brasil, informaçōes referentes à assistência médica evidenciam que a rede hospitalar, assim como a distribuição de leitos, encontra-se concentrada na regiāo Sudeste. A média de leitos $/ 1.000$ habitantes é 3,1 , sendo que na região Sul eleva-se para $3,5 / 1.000$ habitantes. Nessa região, $91,2 \%$ dos leitos são de instituiçóes conveniadas com o SUS ${ }^{19}$. Dos 427 municípios do Rio Grande do Sul, 294 $(69,0 \%)$ possuem hospitais cadastrados no SUS. O Estado apresenta uma média de 2,9 leitos SUS/1.000 habitantes.

As 817.244 internaçốes hospitalares efetuadas no RS em 1995 representaram um coeficiente de 8,6 internaçôes para cada 100 habitantes. Essa é uma morbidade definida à priori, ou seja, as populações já têm definidos o número de AIHs disponíveis, independente do perfil epidemiológico de cada região. Trabalhase com um somatório de internaçóes hospitalares que representam "internaçōes novas" acrescidas das "reinternaçốes". Elas não representam casos "incidentes", na medida em que um mesmo paciente pode estar representado duas vezes. Não foram observadas discrepâncias no padrão regional das internaçōes hospitalares, embora a 1" DRS - a da Região Metropolitana de Porto Alegre - que atende como referência uma parcela significativa da população do Estado - não esteja contemplada proporcionalmente com um número maior de AIHs.

Foram notificados pelo Sistema de Informação de Morbidade Hospitalar 23.974 óbitos hospitalares. A letalidade hospitalar correspondeu à 2,9 óbitos para cada 100 internaçōes.

Estudos de morbi-mortalidade afirmam que os homens morrem mais em todos os grupos etários, porém as mulheres utilizam mais os serviços de saúde. Os dados das AIHs são compatíveis com essa afirmação. Do total de internaçóes, $486.342(59,5 \%)$ referem-se à população do sexo feminino, e as 330.902 restantes $(40,5 \%)$ ao sexo masculino, enquanto os óbitos ocorreram num percentual de $56,7 \%$ nos homens e $43,3 \%$ nas mulheres. A distribuição das AIHs segundo gênero manteve-se semelhante em todas as regionais. Acredita-se que as mulheres apresentem taxas de morbidade mais elevadas "seja pelo uso dos serviços de saúde reprodutivos, seja pela maior capacidade de percepção sobre seu quadro específico de morbidade. Assim, uma das formas de neutralizar esses efeitos sobre a magnitude das taxas de morbidade é decompô-la por sexo e idade"

A distribuição das internações hospitalares no Estado segundo grupo etário evidencia que, $\mathrm{em}$ termos absolutos, o grupo que mais se interna é o de 15 a 49 anos $(45,5 \%)$ embora esse grupo também represente a maior parcela de população. Os menores de um ano representam $8,5 \%$ do total das internaçôes e os pré-escolares, 7,5\%. Na Figura l observa-se os coeficientes de morbidade hospitalar $\left(\mathrm{n}^{\circ}\right.$ de internaçōes dividido pela população) segundo os mesmos grupos etários. Observa-se uma curva em forma de " $U$ ", com um desenho semelhante ao descrito por Medici ${ }^{7}$, mostrando que a concentração de internaçóes hospitalares segundo os grupos etários privilegia o grupo menor de um ano ( $41,5 \%$ para o sexo masculino e $33,1 \%$ para o feminino). Os coeficientes de internação declinam na infầncia, adolescência $\mathrm{e}$ idade adulta para voltar a subir no grupo mais idoso.

A distribuição das AIHs segundo os 17 grupos de causas da CID em 1995 mostra que, no Rio Grande do Sul, o maior percentual de internaçôes correspondeu às doenças respiratórias $(22,9 \%)$, seguindo-se dos partos e suas complicações $(20,8 \%)$, doenças do aparelho circulatório $(12,2 \%)$ e doenças infecciosas $(8,1 \%)$. Contudo, esse padrão não se manteve constante para todas as regionais.

No Brasil, observou-se que as 
Figura 1 - Coeficientes de morbidade hospitalar (SUS) segundo gênero e grupo etário, RS, 1995.

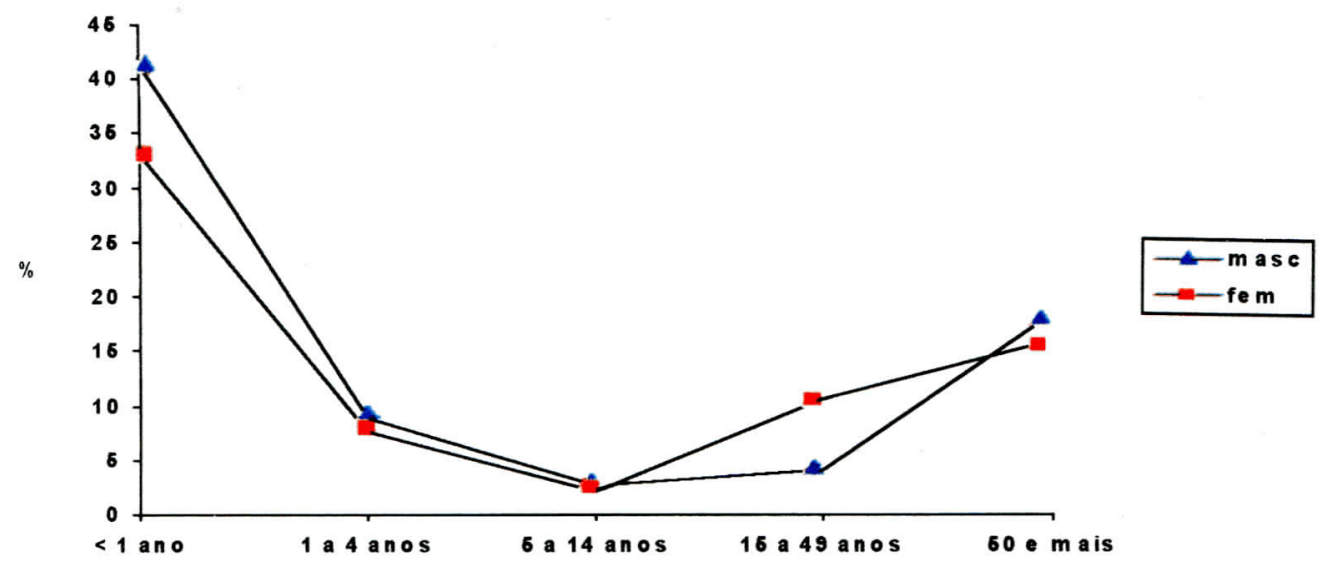

principais causas de internação hốspitalar foram as complicações da gravidez, parto e puerpério, as doenças respiratórias, do aparelho circulatório, infecciosas e parasitárias e do aparelho genitourinário ${ }^{13}$. Portanto, fica evidente no Rio Grande do Sul um perfil de morbidade hospitalar semelhante ao do país como um todo. (Tabela 1).

As diferenças regionais em relação a cada grupo de causa evidenciam discrepâncias

Tabela 1 - Distribuição percentual da morbidade hospitalar (SIH) e mortalidade (SIM e SIH) segundo causas (17 grupos), RS, 1995.

\begin{tabular}{lrrr}
\hline GRUPO DE CAUSAS & Morbidade & \multicolumn{2}{c}{ M ortalidade } \\
& $\%$ & S IM & \multicolumn{1}{c}{ SIH } \\
\hline I. Doenças infecciosas e parasitárias & 8,1 & 2,5 & 9,2 \\
II. Neoplasias & 4,0 & 17,4 & 12,4 \\
III. D. gland. end. nutric. met. e transt. Imunitar. & 2,3 & 4,7 & 3,6 \\
IV. Doenças do sangue e órgãos hem atopoéticos & 0,6 & 0,2 & 0,6 \\
V. Transtornos mentais & 1,9 & 0,3 & 0,0 \\
VI. Doenças do sist. nervoso e dos órgãos dos sentidos & 2,5 & 1,1 & 1,7 \\
VII. Doenças do aparelho circulatório & 12,2 & 34,3 & 27,3 \\
VIII. Doenças do aparelho respiratório & 22,9 & 12,7 & 23,2 \\
IX. Doenças do aparelho digestivo & 7,0 & 4,8 & 7,0 \\
X. Doenças do aparelho geniturinário & 6,0 & 1,5 & 2,7 \\
XI. Complicações da gravidez, parto e puerpério & 20,8 & 0,1 & 0,3 \\
XII. Doenças da pele e tecido celular subcutâneo & 0,8 & 0,1 & 0,3 \\
XIII. Doenças sist. osteomuscular e tec. conjuntivo & 2,0 & 0,3 & 0,6 \\
XIV. Anomalias congênitas & 0,6 & 1,1 & 3,3 \\
XV. Algumas afecções orig. período perinatal & 1,6 & 2,6 & 2,4 \\
XVI Sintomas sinais e afeç̧ões mal definidas & 1,5 & 6,1 & 4,4 \\
XVIII. Causas externas & 5,2 & 10,4 & - \\
\hline TOTAL & 100,0 & 100,0 & 100,0 \\
\hline
\end{tabular}


grosseiras no encaminhamento e nas internações de pacientes. Assim, a la DRS tem arcado com a maioria das internaçóes por todas as causas, destacando-se o elevado percentual de pacientes com neoplasias $(\mathbf{5 4}, \mathbf{1 \%})$, anomalias congênitas $(77,4 \%)$ e causas externas $(64,6 \%)$. Esses percentuais representam mais da metade dos pacientes com essas patologias, quando pela distribuição populacional não deveriam ultrapassar os $30 \%$. Esses dados certamente expressam a concentração de recursos de nível terciário e de maior complexidade tecnológica na capital, assim como a não resolutividade dos serviços locais de saúde, que hipertrofiam as metrópoles com pacientes que, em grande parte, poderiam ser atendidos em seus locais de origem.

A Tabela 2 apresenta os principais motivos de internaçáo segundo sexo e grupos específicos de causas (CID 3 dígitos) no Estado. Pretendia-se delinear um quadro mais acurado das internações, identificando-se doenças que pudessem servir como "marcadoras" e que permitissem apontar problemas e mesmo iniquiidades na distribuição dos serviços de saúde $^{20}$. Porém, o acompanhamento de condições marcadoras como os acidentes vasculares isquêmicos e infarto do miocárdio não foi realizado neste estudo.

As mulheres internaram-se mais para partos na maioria das regióes do Estado. As broncopneumonias também foram uma causa importante de internação. Os procedimentos obstétricos (CID 650 e 669) corresponderam a $17,5 \%$ das causas.

Quanto aos homens, eles estão sendo internados predominantemente por broncopneumonia e obstrução crônica das vias respiratórias. As doenças infecciosas do aparelho respiratório (CID 465, 485 e 486) representaram $10,6 \%$ do total de internaçóes enquanto as doenças pulmonares obstrutivas (CID 492, 493 e 496) corresponderam a 7,3\% da morbidade hospitalar.

Tabela 2 - Principais causas de internações (CID 3 dígitos) segundo gênero, RS, 1995.

\begin{tabular}{clrr}
\hline GÊNERO/CID & CAUSA & $\mathrm{n}$ o & $\%$ \\
\hline Masculino & & & \\
485 & BCP não especificada & 29.507 & 8,9 \\
009 & Infecção intestinal mal definida & 19.563 & 5,9 \\
496 & Obstrução crônica das vias respiratórias & 18.001 & 5,4 \\
428 & Insuficiência cardíaca & 13.506 & 4,1 \\
493 & Asma & 9.192 & 2,8 \\
Subtotal & & 330.902 & \\
Feminino & & & \\
650 & Parto normal & 98.863 & 20,3 \\
669 & Outras compl. trab. parto e parto não compl. & 44.064 & 9,1 \\
485 & BCP org. não específico & 27.738 & 5,7 \\
009 & Infecção intestinal mal definida & 19.599 & 4,0 \\
428 & Insuficiência cardíaca & 18.078 & 3,7 \\
Subtotal & & 486.342 & \\
Total & & & \\
650 & Parto normal & 98.863 & 12,0 \\
485 & BCP microorg. não específico & 57.245 & 7,0 \\
669 & Outras complicações do trabalho de parto e puerpério & 44.064 & 5,4 \\
009 & Infeções intestinais mal definidas & 39.164 & 4,7 \\
428 & Insuficiência cardíaca & 31.584 & 3,8 \\
\hline Total & & 817.244 & \\
\hline McP & & &
\end{tabular}


Além da análise descritiva realizada neste estudo, pretendia-se comparar dados procedentes de dois sistemas de informação distintos, SIM e SIH, para avaliar a fidedignidade de um sistema de registro tão sujeito à críticas como o SIH. O SIM é um registro cuja qualidade tem sido mais avaliada. Comparaçốes similares já haviam sido realizadas em outros estudos ${ }^{13}$. Na Figura 2 observa-se a relação entre as causas de internação e de óbito procedentes de duas fontes: o SIM e o SIH. primeira causa de internação, felizmente não tem repercussão na mortalidade, embora saibase que existe subnotificação da mortalidade materna. Este é, inclusive, mais um banco de dados que permite a busca de informaçōes sobre a mortalidade de mulheres em idade reprodutiva. Interroga-se sobre os baixos percentuais de internação por neoplasias e supóe-se que devam estar sendo codificadas em outras seçōes, devido às complicaçóes ou intercorrências. A terceira causa de internação e primeira de morte -

Figura 2 - Percentual de internações (SIH), mortalidade (SIM e SIH) segundo causa (CID-9), RS,

1995.

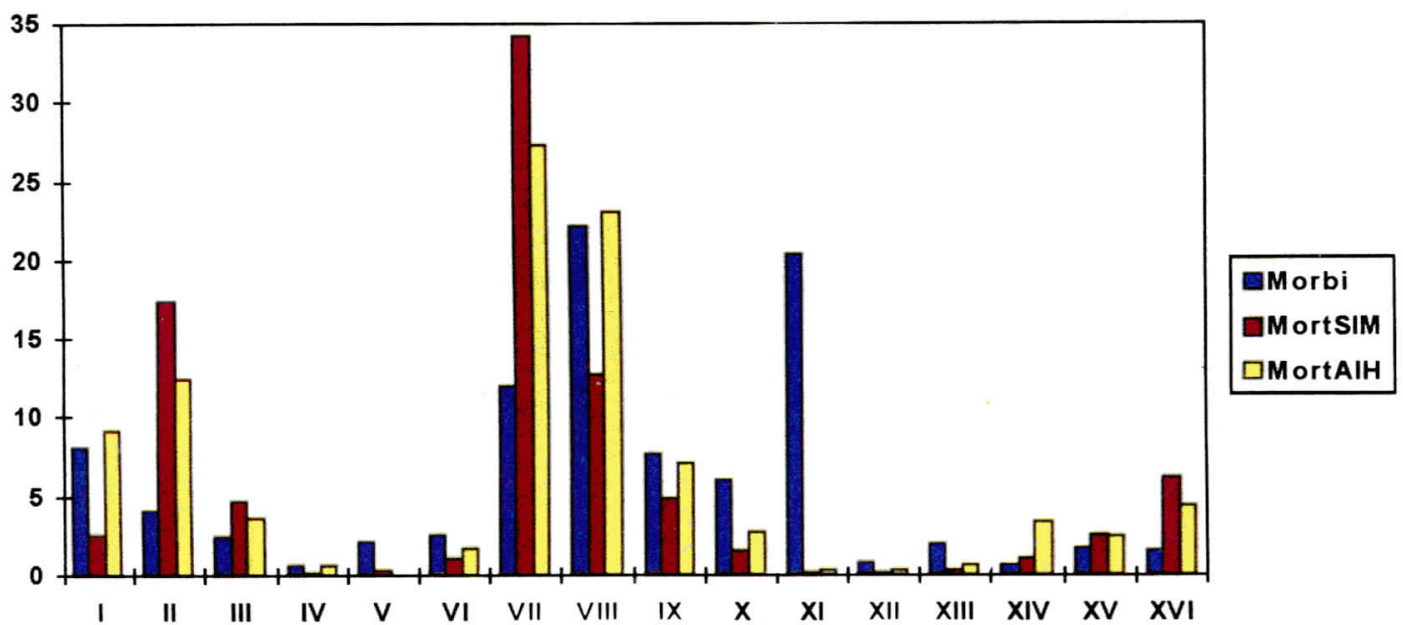

Os registros de mortalidade (SIM) mostraram que, em praticamente todo o Estado, os óbitos obedeceram a seguinte sequência: doenças do aparelho circulatório, neoplasias e doenças do aparelho respiratório. Já as principais causas de internação hospitalar foram: doenças respiratórias, partos e doenças do aparelho circulatório. Quanto à mortalidade hospitalar (SIH) observou-se em primeiro lugar as doenças do aparelho circulatório $(27,3 \%)$, seguindo-se as doenças respiratórias $(23,2 \%)$, neoplasias $(12,4 \%)$ e doenças infecciosas $(9,2 \%)$.

Embora não tenha havido relação direta entre causa de hospitalização e de óbito, como a que apareceu em outros trabalhos ${ }^{13}$, não se pode deixar de apontar certas similaridades $\mathrm{e}$ também aspectos a serem mais esmiuçados. As doenças respiratórias constituem importante causa de internação e de morte. Os partos,

doenças do aparelho circulatório - corresponde a uma situação esperada, embora deva-se salientar que as mortes por doença circulatória poderiam ser postergadas para idades mais avançadas.

\section{Considerações finais}

Neste trabalho ficou explícito que, mesmo com limitaçóes, é possível utilizar para fins epidemiológicos dados secundários procedentes de bancos de dados desenhados originalmente para outros fins. Entre os aspectos positivos do uso do SIH/SUS para o estudo da morbidade hospitalar, inclui-se a sua simplicidade, uma vez que a AIH é um documento de fácil preenchimento $\mathrm{e}$ interpretação; sua ampla cobertura, pois contém os registros de mais de $90 \%$ das internaçóes no 
Estado e seu fácil acesso, através do Programa TAB.

A distribuição da morbidade hospitalar pelo SUS no Estado do Rio Grande do Sul, durante 0 ano de 1995, foi de 8,6 internaçōes para cada 100 pessoas, predominando o sexo feminino e o grupo dos menores de um ano. As principais causas de internação foram: as doenças respiratórias, os partos e as doenças do aparelho circulatório, enquanto as principais causas de mortalidade compreeenderam as circulatórias, neoplasias e respiratórias. A mortalidade pelo SIH mostrou como causas prevalentes de óbito hospitalar as doenças do aparelho circulatório, respiratório e neoplasias. Embora a ordem não seja a mesma, existe uma certa correspondência nas principais causas de internação e morte, mostrando que a qualidade da informação parece não ser das piores. Algumas das características desses dados são semelhantes aos observados em outros locais, enquanto outras situaçóes são peculiares.

Uma das formas de monitorar esse processo seria o acompanhamento de eventos sentinela: por exemplo, acompanhar a mortalidade de agravos em relação aos quais não se espera uma letalidade elevada para, a longo prazo, promover açóes que possam melhorar a qualidade do sistema. Acredita-se que trabalhos como este possam auxiliar indiretamente a melhorar a qualidade $\mathrm{da}$ informação.

Muitos pesquisadores têm comentado sobre a existência de grande volume de dados secundários nas diferentes instituiçōes de pesquisa. Uma das dificuldades para $o$ investigador, porém, é a de reagrupá-los segundo critérios espaciais que permitam potencializar o seu uso. Publica-se, pela primeira vez no Estado, um estudo sistematizado sobre internaçóes hospitalares a partir do SIH/SUS. Acredita-se que esse é um dos pontos altos desta pesquisa e espera-se também que tenha contribuído para melhor esclarecimento da realidade.

\section{Bibliografia}

1. Cordova R.A.; Gusso da, Luna S.V. A Pósgraduação na América Latina: $O$ Caso Brasileiro. Brasília: UNESCO/MEC/ CAPES, 1986.

2. Granda E.; Breilh J. Saúde na Sociedade. São Paulo: Cortez-ABRASCO, 1989.

3. Caminha J.A.; Casarin A; e Bueno I. Indicadores de Saúde. Revista Med. ATM, 9:25-29; 1974.

4. Travassos C.; Fernandes C. e Perez M. Desigualdade Social, Morbidade e Uso de Serviços de Saúde no Brasil; Série Estudos $N^{\circ} 4$ - "Política, Planejamento e Gestão em Saúde". Rio de Janeiro: FIOCRUZ/ENSP, 1995.

5. Castro J.D. A Teoria de Mercado Tradicional e a Saúde. 1995.

6. Carvalho F. M.; Neto A.M.; Paim J.S.; Melo A.M.C.; Azaro M.G.A. Morbidade Referida e Utilização de Consulta Médica em Cinco Populaçóes no Estado da Bahia. Revista Ciência e Cultura. 40(8): 853-858, 1988.

7. Medici A. C. Aspectos Sócio-econômicos da Morbidade no Brasil - Uma Contribuiçáo aos Estudos sobre Populaçáo e Saúde; In: Primeiro Congresso Brasileiro de Epidemiologia, 1990; Campinas, Anais; Rio de Janeiro. ABRASCO, 165-86, 1990.

8. Buss P. M. Assistência Hospitalar no Brasil (1984-1991): Uma Análise Preliminar Baseada no Sistema de Informação Hospitalar do SUS. Informe Epidemiológico do SUS. Brasília: FNS, CENEPI; Ano II, (2): 5-42; 1993.

9. Banco Mundial. Relatório sobre Desenvolvimento Mundial 1993: Investindo em Saúde. Rio de Janeiro: Fundação Getúlio Vargas, 1993.

10. Pinto C.B.; Leal M.C.; Szwarcwald C.L.; Sabroza P., Sevalho G.; Moraes I.H.S. Morbidade Hospitalar na Rede Contratada 
do INAMPS. Dados, FIOCRUZ/ENSP, Ano VI, 1988.

11. Rocha L.E.; Freitas C. U.; Ferreira J. M. N.; Faria C.G; Kumagai M. F. U. Dados Comparativos de Morbidade Hospitalar no Município de Cubatão e do Estado de São Paulo (Brasil). Revista de Saúde Pública. 22 (2): 118-31; São Paulo, 1988.

12. Simeant S. Capacidad Resolutiva de la Demanda de Atencion de Morbilidad a Nível Primário; Chile, 1981. Boletin de la Oficina Sanitaria Panamericana. 125-140, 1984.

13. Yazlle Rocha J. S. e Nogueira J. L. Padróes de Morbidade em Assistência Primária na Região de Ribeirão Preto; SP (Brasil). Revista de Saúde Pública. 19:215-24, 1985.

14. Radaelli S. M. e cols. Demanda de Serviço de Saúde Comunitária na Periferia da Área Metropolitana. Revista de Saúde Pública. 24: 232-40, 1990.

15. Cesar C.L.G. Morbidade Referida e Utilizaçáo de Serviços de Saúde em Localidades Urbanas Brasileiras: Metodologia. Revista de Saúde Pública.
$30(2): 153-60,1996$.

16. Ministério da Saúde. AIH Sistema de Apoio à Entrada de Dados, Manual das Unidades Hospitalares, Instituto Nacional de Assistência Médica da Previdência Social, SUS, Secretaria Nacional da Assistência à Saúde. Brasília, 1995a.

17. Wray N. P. e cols. Using Administrative Databases to Evaluate the Quality of Medical Care: a Conceptual Framework. Soc. Sci. Med. 40 (12): 1707- 15, 1995.

18. Ministério da Saúde. Fundação Nacional de Saúde, Departamento de Informática do SUS. TAB versão l.6a. Programa para realização de tabulaçóes simples - Manual do Usuário. Brasília: Coordenação Geral de Informações em Saúde, 1995b.

19. Viacava F. e Bahia L. Assistência Médica Sanitária - Os serviços de saúde segundo o IBGE. Dados, FIOCRUZ; 3 (30), 1996.

20. Penna M.L.F. e Faerstein E. Coleta de dados ou Sistema de informação? O Método Epidemiológico na Avaliação dos Serviços de Saúde. Cadernos IMS. 1(2): 66-78, 1986. 